\title{
ON AN INEQUALITY OF P. TURAN CONCERNING LEGENDRE POLYNOMIALS
}

\author{
G. SZEGÖ
}

The following remarkable inequality is due to the Hungarian mathematician P. Turán: If $P_{n}(x)$ denotes as usual Legendre's polynomial of the $n$th degree, we have

$$
\Delta_{n}(x)=\left(P_{n}(x)\right)^{2}-P_{n-1}(x) P_{n+1}(x) \geqq 0, \quad n \geqq 1 ;-1 \leqq x \leqq 1,
$$

with equality only for $x= \pm 1$. The purpose of this note is to give several proofs for this theorem different from that of Turán. ${ }^{1}$

1. Proof. The following arrangement is somewhat similar to that of Turán. By using the classical recursion

$$
P_{n+1}(x)=\frac{2 n+1}{n+1} x P_{n}(x)-\frac{n}{n+1} P_{n-1}(x)
$$

we find for the polynomial $\Delta_{n}(x)$ the representation

$$
P_{n}^{2}+\frac{n}{n+1} P_{n-1}^{2}-\frac{2 n+1}{n+1} x P_{n} P_{n-1} \text {. }
$$

This is a quadratic form in $P_{n}$ and $P_{n-1}$ which is positive provided

$$
\frac{n}{n+1}>\left(\frac{n+1 / 2}{n+1} x\right)^{2}, \quad \text { or } \quad|x|<\frac{(n(n+1))^{1 / 2}}{n+1 / 2}=\cos \theta_{0} .
$$

For these $x$ the theorem is already proved. For the remaining $x=\cos \theta$, that is, for $0<\theta \leqq \theta_{0}$, we use Mehler's formula

$$
P_{n}(\cos \theta)=\frac{2}{\pi} \int_{0}^{\theta} \frac{\cos (n+1 / 2) u}{(2(\cos u-\cos \theta))^{1 / 2}} d u
$$

and obtain

$$
\begin{aligned}
& \Delta_{n}(\cos \theta)=\pi^{-2} \int_{0}^{\theta} \int_{0}^{\theta}(\cos u-\cos \theta)^{-1 / 2}(\cos v-\cos \theta)^{-1 / 2} \\
& \cdot\{2 \cos (n+1 / 2) u \cos (n+1 / 2) v-\cos (n-1 / 2) u \cos (n+3 / 2) v \\
& -\cos (n-1 / 2) v \cos (n+3 / 2) u\} d u d v .
\end{aligned}
$$

Presented to the Society, November 30,1946; received by the editors July 11, 1947.

${ }^{1}$ I owe Mr. Turán also some other remarkable properties of the polynomial $\Delta_{n}(x)$. 
The expression in the braces is

$$
\begin{aligned}
\cos (n+1 / 2)(u+v) & +\cos (n+1 / 2)(u-v) \\
& -(1 / 2) \cos [(n-1 / 2) u+(n+3 / 2) v] \\
& -(1 / 2) \cos [(n-1 / 2) u-(n+3 / 2) v] \\
& -(1 / 2) \cos [(n-1 / 2) v+(n+3 / 2) u] \\
& -(1 / 2) \cos [(n-1 / 2) v-(n+3 / 2) u] \\
= & \cos (n+1 / 2)(u+v)(1-\cos (u-v)) \\
& +\cos (n+1 / 2)(u-v)(1-\cos (u+v)),
\end{aligned}
$$

so that $\Delta_{n}>0$ follows provided $(n+1 / 2)|u \pm v| \leqq(n+1 / 2) 2 \theta$ $\leqq(n+1 / 2) 2 \theta_{0} \leqq \pi / 2$. But this is obvious since

$$
\theta_{0}<\frac{\pi}{2} \sin \theta_{0}=\frac{\pi}{2} \frac{1}{2 n+1} .
$$

2. Proof. We expand $\Delta_{n}(x)$ in a finite series of Legendre polynomials which will contain only even terms:

$$
\Delta_{n}(x)=c_{0} P_{0}(x)+c_{1} P_{2}(x)+c_{2} P_{4}(x)+\cdots+c_{n} P_{2 n}(x) .
$$

We show that the coefficients $c_{1}, c_{2}, \cdots, c_{n}$ are negative. Then the minimum will be reached if $P_{m}(x)$ is maximum, that is, for $x=1$. But $\Delta_{n}(1)=0$, thus the inequality will follow.

For this purpose we use a formula due to Adams, Ferrers and F. Neumann for the coefficients of the Legendre expansion of the product of two Legendre polynomials. ${ }^{2}$ It is the simplest to state this formula in form of an integral:

$$
i(a, b, c)=\int_{-1}^{1} P_{a} P_{b} P_{d} d x
$$

$$
\begin{aligned}
& \begin{array}{l}
0 \text { if } a+b+c \text { odd, } \\
0 \text { if } a+b+c \text { even but no triangle with sides }
\end{array} \\
& = \begin{cases}a, b, c \text { exists, } & \\
\frac{2}{2 s+1} \frac{g_{s-a} g_{s-b} g_{o-c}}{g_{\bullet}} & \text { if } a+b+c=2 s, s \text { integer, } \\
& \text { and a triangle with sides } a, b, c \\
\text { exists. }\end{cases}
\end{aligned}
$$

Here

2 See, for instance, E. T. Whittaker and G. N. Watson, Modern analysis, American ed.. 1943, p. 331. 


$$
g_{s}=\frac{1 \cdot 3 \cdots(2 s-1)}{2 \cdot 4 \cdots 2 s} ; \quad g_{0}=1
$$

In our case

$$
\begin{aligned}
\frac{2}{4 \nu+1} c_{\nu} & =i(n, n, 2 \nu)-i(n-1, n+1,2 \nu) \\
& =\frac{2}{2(n+\nu)+1}\left(\frac{g_{\nu} g_{\nu} g_{n-\nu}}{g_{n+\nu}}-\frac{g_{\nu-1} g_{\nu+1} g_{n-\nu}}{g_{n+\nu}}\right), \quad \nu \geqq 1 .
\end{aligned}
$$

$\mathrm{Bu} g_{v} / g_{v-1}$ is increasing so that (12) is negative. This proves the assertion.

The same argument shows that in the expansion

$$
\left(P_{n}(x)\right)^{2}-P_{n-l}(x) P_{n+l}(x)=c_{0} P_{0}(x)+c_{1} P_{2}(x)+\cdots+c_{n} P_{2 n}(x)
$$

the first $l$ coefficients $c_{0}, c_{1}, \cdots, c_{l-1}$ are positive and all the others negative.

3. Proof. Professor Pólya has called my attention to the fact that inequalities of the Turán type occur in the study of entire functions of the form

$$
\sum_{n=0}^{\infty} \frac{u_{n}}{n !} z^{n+r}=e^{-\alpha 22+\beta \varepsilon} z^{r} \prod\left(1+\beta_{n} z\right) e^{-\beta_{n} z}
$$

where $\alpha \geqq 0, \beta$ and $\beta_{n}$ real, $\sum \beta_{n}^{2}$ convergent. These functions, studied by Laguerre, Pólya and others ${ }^{3}$ are (apart from a constant factor) the only ones which are the limits of polynomials with only real roots. A sequence of such polynomials is for instance that of the Jensen polynomials

$$
u_{0}+\left(\begin{array}{l}
n \\
1
\end{array}\right) u_{1} z+\left(\begin{array}{l}
n \\
2
\end{array}\right) u_{2} z^{2}+\cdots+u_{n} z^{n}
$$

which approach the entire function (14) as $n \rightarrow \infty$, provided we replace $z$ by $z / n$. Under the conditions mentioned these polynomials have only real roots. If we denote them by $z_{1}, z_{2}, \cdots, z_{n}$ the inequality $u_{n-1}^{2}-u_{n-2} u_{n} \geqq 0$ is a trivial consequence of the following inequality:

${ }^{3}$ See G. P6lya and I. Schur, Über zwei Arten von Faktorenfolgen in der Theorie der algebraischen Gleichungen, J. Reine Angew. Math. vol. 144 (1914) pp. 89-113; cf. pp. 96-97. 


$$
\left(\frac{z_{1}+z_{2}+\cdots+z_{n}}{n}\right)^{2} \geqq \frac{z_{1} z_{2}+z_{1} z_{3}+\cdots}{\left(\begin{array}{l}
n \\
2
\end{array}\right)} .
$$

The generating function

$$
\sum_{n=0}^{\infty} \frac{P_{n}(x)}{n !} z^{n}=e^{x z} J_{0}\left(\left(1-x^{2}\right)^{1 / 2} z\right)
$$

shows, in view of well known properties of the Bessel function $J_{0}$, that the conditions mentioned above are indeed satisfied. This furnishes the theorem.

Taking into account the identities

$$
\begin{array}{ll}
\sum_{n=0}^{\infty} \frac{P_{n}^{(\lambda)}(x)}{P_{n}^{(\lambda)}(1)} \frac{z^{n}}{n !}=2^{\lambda-1 / 2} \Gamma(\lambda+1 / 2) e^{x z}\left(\left(1-x^{2}\right)^{1 / 2} z\right)^{1 / 2-\lambda} & \lambda>-1 / 2, \\
\sum_{n=0}^{\infty} \frac{L_{n}^{(\alpha)}(x)}{L_{n}^{(\alpha)}(0)} \frac{z^{n}}{n !}=\Gamma(\alpha+1) e^{z}(x z)^{-\alpha / 2} J_{\alpha}\left(2(x z)^{1 / 2}\right), & \alpha>-1, \\
\sum_{n=0}^{\infty} \frac{H_{n}(x)}{n !} z^{n}=e^{2 x z-z^{2}} &
\end{array}
$$

where $P_{n}^{(\lambda)}, L_{n}^{(\alpha)}, H_{n}$ denote the ultraspherical, Laguerre and Hermite polynomials, respectively, we conclude the analogous inequalities for these polynomials. ${ }^{4}$

4. Proof. Finally we can avoid the use of transcendental functions and work only with the Jensen polynomial (15). It can be written in this particular case in the following form:

$$
\begin{aligned}
P_{0}(x)+\left(\begin{array}{l}
n \\
1
\end{array}\right) P_{1}(x) z & +\left(\begin{array}{l}
n \\
2
\end{array}\right) P_{2}(x) z^{2}+\cdots+P_{n}(x) z^{n} \\
& =\left(1+2 x z+z^{2}\right)^{n / 2} P_{n}\left(\frac{1+x z}{\left(1+2 x z+z^{2}\right)^{1 / 2}}\right) .
\end{aligned}
$$

This identity can be shown by the ordinary generating function or by the generating function (17) or by the first integral of Laplace. ${ }^{5}$ Now

4 We follow the notation of G. Szegö, Orthogonal polynomials, Amer. Math. Soc. Colloquium Publications, vol. 23, 1939, pp. 80, 98, 102. The inequality for the Hermite polynomials was pointed out to me by $P$. Turán.

${ }^{6}$ See G. Szegö, loc. cit. p. 87. 
let $x$ be fixed, $-1<x<1$. We obtain for the roots of the polynomial (19) in $z$ the condition

$$
\frac{1+x z}{\left(1+2 x z+z^{2}\right)^{1 / 2}}=x_{\nu}
$$

where $x_{\nu}$ denotes a root of $P_{n}$. Or

$$
z=\frac{x\left(x_{\nu}^{2}-1\right) \pm x_{\nu}\left(\left(1-x_{v}^{2}\right)\left(1-x^{2}\right)\right)^{1 / 2}}{x^{2}-x_{\nu}^{2}}
$$

thus the roots in $z$ are all real. Using the trivial inequality (16) the assertion follows.

STANFORD UNIVERSITY

\section{NOTE ON THE EIGENVALUES OF THE STURM-LIOUVILLE} DIFFERENTIAL EQUATION

GERALD FREILICH

In discussing eigenvalues and eigenfunctions of the Sturm-Liouville differential equation

$$
L(u)+\lambda \rho u=0, \quad L(u)=\left(p u^{\prime}\right)^{\prime}-q u,
$$

with

$$
\left.\begin{array}{rl}
p(x) & \geqq m>0 \\
q(x) & \geqq 0 \\
\beta & \geqq \rho(x) \geqq \alpha>0
\end{array}\right\} \quad \text { for } a \leqq x \leqq b, \text { and for some } \alpha, \beta, \text { and } m,
$$

and the boundary conditions

$$
u(a)=c_{1} u(b), \quad u^{\prime}(a)=c_{2} u^{\prime}(b), \quad c_{1} c_{2} p(a)=p(b),
$$

we find that we can represent our eigenfunctions as unit normals in the directions of the principal axes of an ellipsoid in function space. We define our function space $F$ as the set of all functions $v(x)$, $a \leqq x \leqq b$, which satisfy the boundary conditions of the Sturm-Liouville equation. The origin of our space will be the function $u(x)=0$. We can now metrize $F$ by defining our inner product $(u, v)$ for

Received by the editors June 26, 1947. 